\title{
Modelos Matemáticos, Simulação da Produção e Índice Tecnológico de Municípios do Rio de Janeiro
}

\author{
R. M. AQUINO ${ }^{1 *}$, C. A. R. VERA-TUDELA ${ }^{2}$, J. C. A. BASTOS ${ }^{3}$, \\ S. D. VENTURA ${ }^{4}$ e R. F. DE OLIVEIRA ${ }^{5}$
}

Recebido em 31 de julho de 2018 / Aceito em 11 de janeiro de 2020

\begin{abstract}
RESUMO. Neste trabalho foram desenvolvidos modelos matemáticos que permitem simular a produção de mel de uma determinada região. Esses modelos são baseados em equações diferenciais ordinárias e tiveram como ponto de partida o modelo neoclássico de Lucas [23] para o crescimento macroeconômico. Na determinação dos parâmetros dos modelos foram utilizados métodos numéricos de otimização não linear. Como resultado da solução numérica do modelo, foram simuladas a produção de mel de quatro municípios. Obteve-se, ainda, o índice de tecnologia aplicado à produção de mel de cada localidade, por um período de onze anos. Por meio desse índice é possível ordenar as municipalidades em termos de aplicação de tecnologia. Essa é uma das razões desse índice ser um indicador de vital importância para a gestão do agronegócio.
\end{abstract}

Palavras-chave: modelo de Lucas, modelos matemáticos, produção de mel.

\section{INTRODUÇÃO}

O presente trabalho tem como foco a apicultura, mais especificamente o desenvolvimento de uma ferramenta matemática que permita modelar a produção melífera em uma determinada região, e seja capaz de fornecer ao gestor do agronegócio um indicador fundamental para a tomada de decisões que visem ao aprimoramento dos fatores produtivos. Os modelos aqui desenvolvidos analisam a apicultura sob a ótica puramente econômica, tendo como base o Modelo de Lucas para

\footnotetext{
*Autor correspondente: Renato Machado Aquino - E-mail: renaqui60@ gmail.com

${ }^{1}$ Departamento de Matemática, ICE, Universidade Federal Rural do Rio de Janeiro, Caixa Postal, 74517, 23897-970, Seropédica, RJ, Brasil - E-mail: renaqui60@ gmail.com https://orcid.org/000-0001-5116-8928

2 Departamento de Matemática, ICE, Universidade Federal Rural do Rio de Janeiro, Caixa Postal, 74517, 23897-970, Seropédica, RJ, Brasil. - E-mail: candres@ufrrj.br https://orcid.org/0000-0001-5855-8611

${ }^{3}$ Faculdade de Economia, Universidade Federal Fluminense, Campus Gragoatá, São Domingos, 24210-201, Niterói, RJ, Brasil. - E-mail: juliobastos@globo.com https://orcid.org/000-0003-3201-7555

${ }^{4}$ Departamento de Matemática, ICE, Universidade Federal Rural do Rio de Janeiro, Caixa Postal, 74517, 23897-970, Seropédica, RJ, Brasil. - E-mail: ventura@ufrrj.br https://orcid.org/ 0000-0001-9166-1856

5 Departamento de Matemática, ICE, Universidade Federal Rural do Rio de Janeiro, Caixa Postal, 74517, 23897-970, Seropédica, RJ, Brasil. - E-mail: rosane@ufrrj.br https://orcid.org/ 0000-0003-0495-7339
} 
o desenvolvimento econômico de longo prazo [23]. Foram construídos a partir da modelagem de dados de produção de mel em municípios do Rio de Janeiro, tomados entre os anos de 2001 e 2011, o que permitiu a obtenção de índice de tecnologia [23] da produção de mel relativo a uma localidade. Tal parâmetro revela-se um subsídio importante que permite um gerenciamento mais efetivo, por exemplo, da distribuição de incentivos, com vistas à racionalização da produção local.

Dada a sua abrangência, pode-se aplicar a ferramenta desenvolvida a outras modalidades de produção e, obedecidas as restrições impostas poderá ser usada em outras regiões, contribuindo para ganhos operacionais do processo e maior desenvolvimento.

Apesar da multiplicidade de fatores que intervêm na produção melífera, foram utilizadas apenas duas variáveis no processo de modelagem: a produção e o capital humano, sendo este último fator, ao que tudo indica, o principal responsável pela redução do número de variáveis na modelagem. Os modelos construídos são baseados em sistemas de equações diferenciais não lineares. Foram desenvolvidos algoritmos na linguagem Fortran [5], com o objetivo de ajustar os parâmetros dos sistemas de equações, de forma que as suas soluções estivessem dentro de uma margem de erro pré-definida dos dados reais de produção de mel. Para o ajuste, utilizou-se o método dos mínimos quadrados não lineares.

Na próxima seção, será feita uma breve revisão da literatura, localizando a nossa contribuição dentro do contexto das publicações sobre apicultura. A seção 3 dará suporte teórico ao artigo, tanto do ponto de vista econômico quanto matemático, localizando-se na seção 4 o método utilizado na solução dos sistemas de equações que tratam do modelo desenvolvido e do aporte computacional empregado. As duas últimas seções mostram alguns resultados obtidos, e nelas são discutidas as contribuições desta pesquisa.

\section{REVISÃO DA LITERATURA}

De acordo com Gonçalves [13], são diversas as linhas de investigação que têm a apicultura como seu objeto, desde técnicas de manejo até a biologia das abelhas. Dentro desse contexto, modelos matemáticos vêm sendo utilizados nessa área desde longa data. Por exemplo, o número de crias em uma colmeia ao longo do ano foi modelado por Rowland e McLellan já em 1982. No mesmo trabalho, os autores mencionam um modelo mais antigo, cuja complexidade eles haviam aprimorado [28]. Em períodos mais recentes, pode-se encontrar o uso tanto de modelos estocásticos quanto determinísticos, em particular aqueles baseados em equações diferenciais, para modelar, por exemplo, a dinâmica e estrutura populacional de colmeias $[6,10,20,33]$; sanidade apícola $[8,18,30]$ e o manejo apícola [11].

Na perspectiva econômica, vários são os trabalhos encontrados na literatura que utilizam modelos matemáticos na avaliação da viabilidade do agronegócio apícola em determinada região. São esses os modelos que mais se adequam ao contexto de aplicação deste trabalho, qual seja, o que relaciona variáveis econômicas e produção de mel em certa localidade, ao longo do tempo. 
Para estimar o potencial econômico da apicultura em certa região, por exemplo, várias pesquisas ocuparam-se de avaliar o índice de tecnologia dessa modalidade produtiva, bem como fatores que o balizavam $[4,19,21,25,26]$. Em geral, esses trabalhos fizeram um levantamento socioeconômico dos apicultores de certa localidade, baseando-se em alguma técnica de amostragem. Através de métodos de regressão, buscavam determinar a relação entre os fatores relacionados à produção e o índice de tecnologia já calculado. Como tais processos demandavam de um levantamento prévio de informações a respeito dos apicultores e da produção, os pesquisadores viram-se na dependência de informações nem sempre disponíveis, obrigando-os, como alternativa, a fazerem eles mesmos tal levantamento. Trabalhos de grande escala tornavam-se assim proibitivos, exceto se existissem informações que disponibilizassem esses dados, como censos. Além disso, a implementação do método e os testes de significância necessários podem complicar sobremaneira o seu uso (problemas de autocorrelação, heterocedasticidade e normalidade, dentre outros).

Um exemplo de modelagem de interesse ao nosso caso foi a desenvolvida na Turquia, aplicada à produção de mel e cera [9]. Com base numa função de Cobb-Douglas, utilizou-se, para esses dois produtos apícolas, a análise de séries temporais de valores de produção entre 1936 e 2012. Após linearizarem as funções de produção propostas, os autores aplicaram o método dos mínimos quadrados e ajustaram os parâmetros, supondo que os resíduos pudessem ser modelados pelo ARMA (Modelo Auto-regressivo de Médias Móveis). Ao final, foram relacionados os parâmetros encontrados para ambas as produções.

Os modelos matemáticos desenvolvidos no presente trabalho permitem avaliar o índice tecnológico de certa localidade, a partir apenas de dados de produção de mel ao longo do tempo. Conhecidos os dados de produção, tais modelos revelam-se vantajosos. No caso, foi modelada a produção de mel de municípios do estado do Rio de Janeiro, também utilizando uma função de Cobb-Douglas. As séries temporais utilizadas compreendem períodos de onze anos entre 2001 e 2011. Os parâmetros avaliados são basicamente os mesmos do estudo na Turquia, como o índice de tecnologia. As metodologias empregadas, no entanto, diferem bastante: as ferramentas matemáticas aqui utilizadas são sistemas de equações diferenciais ordinárias, sendo que os parâmetros são estimados lançando-se mão de técnicas numéricas de otimização.

\section{OS MODELOS}

Esta seção será dividida em duas partes. Na primeira será feita uma breve descrição do modelo econômico que serviu como base aos modelos construídos no presente trabalho. Na segunda procede-se à construção dos modelos propriamente ditos.

\subsection{O Modelo de Lucas para o Crescimento Econômico}

O presente trabalho teve como base o modelo para o crescimento macroeconômico de Lucas [23]. Esse modelo tem como substrato a ideia de que o crescimento tem como motores forças inerentes ao processo produtivo. De acordo com a referência [29], não são tecnologias exógenas sobre 
as quais o mercado não tem nenhum controle que comandam o crescimento, mas sim forças econômicas endógenas aos sistemas de mercado descentralizados.

Algumas concepções econômicas pregavam que o capital humano ${ }^{1}$ é um fator gerador de crescimento, como a tecnologia e o capital físico, acumulável como este último e tendo forte determinação exógena. Ao contrário, para Lucas [23], o capital humano é um fator endógeno ao processo produtivo, que produziria efeitos positivos para o crescimento econômico. O indivíduo acumularia capital humano e competências de maneira análoga à aquisição de conhecimento técnico: ele utilizaria o conhecimento já existente para adquirir novos conhecimentos e, portanto, mais capital humano. Além disso, o capital humano pode ser apropriado pelos indivíduos [16].

Os pressupostos do modelo de Lucas são os seguintes:

(i) A economia em que o modelo se baseia é fechada à competitividade externa.

(ii) Os agentes econômicos agiriam segundo a Hipótese das Expectativas Racionais ${ }^{2}$ e a tecnologia produziria retornos constantes ${ }^{3}$.

(iii) Em determinado instante, a mão de obra apresenta uma taxa de crescimento exógena.

(iv) A economia gira em torno de apenas um produto.

(v) O capital é proporcional ao número de indivíduos produtivos.

(vi) Por hipótese, a economia trabalhada é fechada.

Dessa forma, como condição de equilíbrio macroeconômico, impõe-se que a produção agregada é igual à demanda agregada [17]. A seguinte equação diferencial descreve essa condição de equilíbrio [23],

$$
N(t) c(t)+\frac{d K(t)}{d t}=A(t) K^{\alpha}(t) N^{1-\alpha}(t),
$$

onde o lado esquerdo da igualdade corresponde à Demanda Agregada e o lado direito da igualdade corresponde à Produção Agregada. Também, $N(t)$ é a mão de obra disponível no instante $t$; $c(t)$ é o consumo per capita; $K(t)$ é o capital físico; $A(t)>0$ é o índice de tecnologia aplicado à produção, todos relativos ao instante $t$. Por fim, $\alpha$ é o coeficiente de elasticidade da produção em relação ao capital físico.

Considerando o capital humano um fator determinante na produção, se $h(t)$ é o capital humano per capita, sendo o mesmo para todos os trabalhadores no instante $t$, valerá a igualdade,

\footnotetext{
${ }^{1}$ Neste trabalho, o capital humano será considerado o conjunto de habilidades apresentadas por um trabalhador em certo instante.

${ }^{2}$ Segundo Dathein [7], os agentes econômicos satisfazem a Hipótese das Expectativas Racionais quando são formadas suas expectativas e estratégias de ação segundo as informações disponíveis, não havendo margem para erros sistemáticos. Essa hipótese é relaxada para o Modelo B, apresentado mais adiante.

${ }^{3}$ Retornos constantes de tecnologia significam que os ganhos de certa economia são diretamente proporcionais à sua tecnologia.
} 
$H(t)=h(t) N(t)$, onde $H(t)$ é o capital humano total presente nessa economia, nesse instante. Dessa forma, a equação 3.1 pode ser reescrita como [23],

$$
N(t) c(t)+\frac{d K(t)}{d t}=A K^{\alpha}(t)[u(t) H(t)]^{1-\alpha} h_{\alpha}^{\gamma}(t),
$$

onde $u(t)$ é a porcentagem da força de trabalho empregada na produção no instante $t$, enquanto $h_{\alpha}^{\gamma}(t)$ descreve os efeitos do capital humano médio sobre os fatores de produção, que independe de decisões individuais de acumulação de capital. Esse fator mede os efeitos externos do capital humano, todos no instante $t$. Note-se que, na equação anterior, o índice de tecnologia $A$ é considerado constante no curto e no médio prazo [23].

Para a variação do capital humano, o mesmo autor propõe a equação 3.3

$$
\frac{d h(t)}{d t}=G(1-u(t)) h^{\gamma}(t)
$$

em que $G$ é uma função crescente com $G(0)=0$ e $1-u(t)$ é a porcentagem da força de trabalho aplicada na formação de mais capital humano, no instante $t$. Lucas observa que, se $\gamma<1$, existem retornos decrescentes à acumulação de capital humano. Isso implicaria que a taxa de acumulação de capital humano tenderia a zero, não importando o esforço dedicado à sua acumulação. Devido a isso, ele resgata o modelo de Uzawa [31], onde esse autor trabalha com uma função $G$ que é linear em $1-u(t)$ e, na qual, $\gamma=1$. Esse modelo possui crescimento sustentado da renda per capita derivado apenas de uma acumulação endógena do capital humano. Portanto, a variação do capital humano no modelo de Lucas fica descrita pela equação 3.4

$$
\frac{d h(t)}{d t}=B(1-u(t)) h(t)
$$

na qual $B>0$ é o índice de tecnologia na produção de capital humano.

\subsection{A Construção dos Modelos}

O modelo que será proposto a seguir tem os mesmos pressupostos do Modelo de Lucas, apresentado na seção anterior. Como esses modelos são originais, será feita a dedução dos mesmos. As equações utilizadas serão as equações 3.2 e 3.4 .

É importante ressaltar que as equações do Modelo de Lucas foram escolhidas como base para o trabalho aqui desenvolvido pelas seguintes razões: conseguem descrever a evolução de economias ao longo do tempo e, com algumas simplificações, é possível chegar a uma equação que tenha como variável a produção. Outra razão para a adoção dessas equações foi considerar a importância do capital humano para a evolução econômica. Por outro lado, segundo Lorenzón [22], toda a produção de mel do Estado do Rio de Janeiro é consumida no próprio estado satisfazendo, portanto, um dos principais pressupostos do Modelo de Lucas que estabelece que a economia em foco é fechada. Outrossim, apesar do modelo de Lucas ter como função precípua a descrição de uma economia no sentido macroeconômico, ele é microfundamentado [1]. Isso significa que ele também é útil para descrever fenômenos microeconômicos, como no caso deste estudo. 
O modelo empregado no presente trabalho tem como foco a produção, ou seja, é baseado na oferta, conforme [24]. Portanto, a parcela referente ao consumo na equação 3.2 pode ser desconsiderada. Além disso, o fator $h_{\alpha}(t)$ no segundo membro da mesma equação refere-se ao efeito do capital humano sobre a economia como um todo. Da mesma forma que [31], para efeito de simplificação vamos assumir $\gamma=0$, o que produzirá $h_{\alpha}^{\gamma}(t)=1$.

As equações 3.2 e 3.4 ficam da forma seguinte,

$$
\begin{gathered}
\frac{d K}{d t}=A K^{\alpha}[u H]^{1-\alpha}, \\
\frac{d h}{d t}=B(1-u) h,
\end{gathered}
$$

onde a variável $t$ foi omitida por questão de simplicidade. Esse procedimento será adotado daqui para a frente.

Por outro lado, como a apicultura é uma atividade não intensiva de mão de obra, por ser baseada em pequenos produtores e na agricultura familiar, pode-se considerar que $\frac{d N}{d t}=0$, ou seja, a variação da mão de obra é desprezível ao longo do tempo [12]. Como $H=h N$, a variável $h$ pode ser explicitada como: $h=\frac{H}{N}$. Portanto, ao se substituir $h$ na equação 3.6, obtém-se a seguinte igualdade:

$$
\frac{1}{N}\left(\frac{d H}{d t}\right)=B(1-u) \frac{H}{N}
$$

Usando o fato de que $N$ é uma constante não nula, ela pode ser cancelada nos dois membros da equação 3.7 obtendo-se:

$$
\frac{d H}{d t}=B(1-u) H
$$

O modelo que será utilizado aqui será deduzido a partir das equações 3.5 e 3.8 .

Seja $P=P(t)$ a produção no instante $t$. De acordo com Varian [32], o segundo membro da equação 3.5 é a função de produção de Cobb-Douglas $P(t)=A K^{\alpha}(u H)^{1-\alpha}$, que pode ser reescrita da seguinte forma:

$$
P=A K^{\alpha} u^{1-\alpha} H^{1-\alpha}
$$

Expressando $K$ em função de $P$ na equação 3.9 e derivando ambos os membros da igualdade obtida em função de $t$, obtém-se:

$$
\frac{d K}{d t}=A^{-\frac{1}{\alpha}} \frac{d P^{\frac{1}{\alpha}}}{d t} u^{\frac{\alpha-1}{\alpha}} H^{\frac{\alpha-1}{\alpha}}+A^{-\frac{1}{\alpha}} P^{\frac{1}{\alpha}} \frac{d u^{\frac{\alpha-1}{\alpha}}}{d t} H^{\frac{\alpha-1}{\alpha}}+A^{-\frac{1}{\alpha}} P^{\frac{1}{\alpha}} u^{\frac{\alpha-1}{\alpha}} \frac{d H^{\frac{\alpha-1}{\alpha}}}{d t},
$$

onde foi utilizado que o índice de tecnologia $A$ é constante.

Após desenvolver as derivadas no segundo membro da equação 3.10 e lembrando que das equações 3.5 e 3.9 tem-se que $\frac{d K}{d t}=P$, pode-se escrever:

$$
\begin{aligned}
P=\frac{1}{\alpha} A^{-\frac{1}{\alpha}} \frac{d P}{d t} P^{\frac{1}{\alpha}-1} u^{\frac{\alpha-1}{\alpha}} H^{\frac{\alpha-1}{\alpha}}+\left(\frac{\alpha-1}{\alpha}\right) A^{-\frac{1}{\alpha}} P^{\frac{1}{\alpha}} \frac{d u}{d t} u^{\frac{-1}{\alpha}} H^{\frac{\alpha-1}{\alpha}}+ & \\
& \left(\frac{\alpha-1}{\alpha}\right) A^{-\frac{1}{\alpha}} P^{\frac{1}{\alpha}} u^{\frac{\alpha-1}{\alpha}} \frac{d H}{d t} H^{\frac{-1}{\alpha}}
\end{aligned}
$$


Multiplicando-se os dois membros da equação 3.11 por $\alpha P^{l-\frac{1}{\alpha}} A^{\frac{1}{\alpha}} u^{\frac{l-\alpha}{\alpha}} H^{\frac{l-\alpha}{\alpha}}$ e efetuando as simplificações, a seguinte igualdade é obtida:

$$
\frac{d P}{d t}+(\alpha-1) \frac{P}{H} \frac{d H}{d t}+(\alpha-1) \frac{P}{u} \frac{d u}{d t}=\alpha P^{\frac{2 \alpha-1}{\alpha}} A^{\frac{1}{\alpha}}(u H)^{\frac{1-\alpha}{\alpha}} .
$$

Considerando $H_{P}$ o capital humano utilizado na produção de mel, como $u$ é a porcentagem do capital humano utilizada na produção, vale que $u=\frac{H_{P}}{H}$. Se os dois membros dessa igualdade forem derivados, segue que:

$$
\frac{d u}{d t}=\frac{1}{H} \frac{d H_{P}}{d t}-\frac{H_{P}}{H^{2}} \frac{d H}{d t} .
$$

Como a apicultura é desenvolvida ainda com dificuldade no país e basicamente por pequenos e médios apicultores, o capital humano aplicado à produção pode ser considerado constante, o que significa que $\frac{d H_{P}}{d t}$ pode ser aceito como nulo. Portanto, segue da equação 3.13 que:

$$
\frac{1}{H} \frac{d H}{d t}=-\frac{1}{u} \frac{d u}{d t} .
$$

Das equações 3.12 e 3.14 pode ser deduzida a igualdade,

$$
\frac{d P}{d t}=\alpha A^{\frac{1}{\alpha}}(u H)^{\frac{1-\alpha}{\alpha}} P^{\frac{2 \alpha-1}{\alpha}},
$$

que é a equação que relaciona produção e capital humano a ser adotada.

Escrevendo as equações 3.15 e 3.8 em um mesmo sistema:

$$
\left\{\begin{array}{l}
\frac{d P}{d t}=\alpha A^{\frac{1}{\alpha}}(u H)^{\frac{1-\alpha}{\alpha}} P^{\frac{2 \alpha-1}{\alpha}} \\
\frac{d H}{d t}=B(1-u) H
\end{array}\right.
$$

que será um dos modelos trabalhados aqui.

Para que se leve em consideração, na equação 3.16, a possibilidade de a produção $P$ ser decrescente em certo intervalo de tempo, será introduzida a produção média, $P_{\text {med }}$ no instante $t$, definida como:

$$
P_{\text {med }}(t)=(-1)^{\mu} \frac{P(t)}{N_{o p}},
$$

em que $N_{o p}$ é o número médio de trabalhadores na produção no instante $t$, sendo $\mu$ par, se a produção for crescente no instante $t$ e ímpar, se a produção for decrescente em dado instante.

Introduzindo a variável $P_{\text {med }}$ como fator no segundo membro da equação 3.16 de produção, após efetuar-se os cálculos devidos obtém-se o outro modelo matemático a ser trabalhado. O mesmo assume a seguinte forma:

$$
\left\{\begin{array}{l}
\frac{d P}{d t}=\frac{\alpha(-1)^{\mu} A^{\frac{1}{\alpha}}(u H)^{\frac{1-\alpha}{\alpha}} P_{\text {med }}^{\frac{3 \alpha-1}{\alpha}}}{N_{o p}} \\
\frac{d H}{d t}=B(1-u) H
\end{array} .\right.
$$

É importante ressaltar que todas as grandezas presentes nas equações 3.16 e 3.18 serão trabalhadas em dólares. Esse procedimento garante a homogeneidade dimensional das mesmas e elimina possíveis efeitos de inflação. Outro ponto a ser destacado é que, daqui para a frente, as equações 3.16 serão denominadas Modelo A. Por seu turno, as equações 3.18 serão o Modelo B. 


\section{METODOLOGIA EMPREGADA}

Nas seções seguintes, será apresentada a metodologia empregada na solução dos modelos matemáticos. Na primeira delas será descrito o método utilizado na busca de soluções dos mesmos, assim como será apresentado o solver utilizado nessa tarefa. A segunda delas descreve, em detalhe, o processo de validação dos modelos.

\subsection{A Busca por Soluções dos Modelos}

Na busca do melhor ajuste para os modelos com equações 3.16 e 3.18, utilizou-se o método dos mínimos quadrados não linear. Nesse caso, dadas condições iniciais para a produção e capital humano, foram procuradas soluções aproximadas para os problemas de valor inicial correspondentes. Essas soluções deveriam ser tais que, somas do tipo:

$$
\sum_{i=1}^{i_{\max }}\left\|x\left(t_{i}, m\right)-\hat{x}_{i}\right\|_{2}^{2},
$$

devessem ser menores que uma margem de erro pré-estabelecida, para um determinado vetor $m$ de parâmetros dos modelos. No caso, $x(t, m)=\left(x_{l}(t, m), x_{2}(t, m)\right)$ é o vetor cuja primeira componente é a produção de mel e a segunda componente é o capital humano para valores da variável $t$ entre $t_{\min }$ e $t_{\max }$. Mais ainda, $\hat{x}_{i}=\left(\hat{x}_{1, i}, \hat{x}_{2, i}\right)$ para $1 \leq i \leq i_{\text {max }}$ são vetores nos quais a primeira componente e a segunda componentes são valores conhecidos da produção e do capital humano nos instantest $t_{i}$.

O algoritmo utilizado para solucionar o problema de mínimos quadrados não lineares, equação 4.1, baseia-se em regiões de confiança, sendo livre de derivadas. Ele é denominado BOBYQA (Bounded Optimization by Quadratic Approximations), tendo sido programado em Fortran 77 [27]. Trata-se da extensão de um programa anterior desenvolvido pelo mesmo autor, que modificou este último para adaptá-lo a problemas com restrições do tipo caixa.

Algumas características básicas do BOBYQA são as seguintes [2]:

(a) O menor valor $r$ entre o raio da região de confiança e o raio do conjunto de pontos interpoladores é escolhido. Isso é feito de modo que os pontos interpoladores estejam suficientemente distantes, a fim de evitar a influência de erros nos valores da função objetivo. Portanto, o critério de mudança do raio da região de confiança em passos consecutivos do algoritmo obedece a uma estratégia refinada.

(b) O algoritmo tenta construir modelos quadráticos apropriados utilizando menos dados que os algoritmos tradicionais baseados em região de confiança. Supondo que um modelo quadrático $m_{k}$ tenha $\frac{k(k+1)}{2}$ coeficientes a determinar, esse número ficaria muito elevado para problemas com dimensão alta.

(c) o BOBYQA emprega uma adaptação do método do gradiente conjugado para regiões de confiança, a fim de minimizar a forma quadrática nessas regiões. 
(d) o BOBYQA é aplicado a funções objetivo do tipo "black box", isto é, funções cujos valores são resultados de uma subrotina. Não é exigida a existência de derivadas para função objetivo.

O algoritmo desenvolvido neste trabalho realiza o ajuste dos parâmetros dos sistemas de equações diferenciais que compõem os modelos A e B. Esse algoritmo foi escrito na linguagem Fortran 95 e compilado usando-se o software de código aberto Gfortran.

\subsection{A Validação dos Modelos}

A validação dos modelos teve duas etapas:

\subsubsection{Etapa 1}

Nesta etapa fez-se o ajuste do Modelo B, com atribuição de valores aleatórios aos parâmetros $\alpha, A, B, u$ e $N_{o p}$, dentro dos intervalos expressos na equação 4.2. Tais valores foram gerados no Matlab ${ }^{\mathrm{TM}}$ sendo utilizadas apenas funções básicas do ambiente como, por exemplo, a função rand. Esta função gera números uniformemente distribuídos no intervalo $[0,1]$. Atribuídos os valores aos parâmetros, efetuou-se a resolução do sistema de equações correspondente, do qual foram obtidos dados gedanken ${ }^{4}$ de produção e capital humanos para os 11 anos abordados neste estudo. Esses valores foram gerados sem ruído. No caso, foram obtidos 73 conjuntos de dados simulados. É importante observar que o intervalo máximo de 11 anos foi escolhido, a princípio, pela estabilidade apresentada, em testes preliminares, pelo algoritmo do Gradiente Conjugado não linear nessa margem de anos. Prefiriu-se, então, manter a mesma margem de anos para o BOBYQA.

Após serem obtidos os 73 conjuntos de dados simulados (gedanken), o algoritmo correspondente ao Modelo B foi aplicado a esses conjuntos de dados. Efetuou-se, então, a recuperação dos cinco parâmetros $\alpha, A, B, u$ e $N_{o p}$. Não foram adicionados ruídos a essas recuperações, sendo selecionado desses conjuntos, pelo interesse desse trabalho, o que melhor forneceu o índice de tecnologia A. Chame-se atenção ao fato de tal parâmetro não ter se mostrado um parâmetro de fácil recuperação ${ }^{5}$. Os valores correspondentes de capital humano, desse conjunto que forneceu a melhor recuperação do parâmetro $\mathrm{A}$, foram utilizados na validação dos modelos $\mathrm{A}$ e $\mathrm{B}^{6}$. Isso ocorreu quando da verificação de seus ajustes aos dados reais de produção.

\footnotetext{
${ }^{4}$ Dados gedanken significam, no presente contexto, dados simulados.

${ }^{5}$ A dificuldade de recuperação do parâmetro A sugeriu, também, que o critério adotado poderia possibilitar boas recuperações de alguns dos demais parâmetros, quando dos ajustes feitos utilizando-se dados reais.

${ }^{6}$ É importante lembrar que a segunda etapa, que consiste da recuperação dos parâmetros $\alpha, A, B, u$ que melhor ajustem os modelos a um conjunto dado de valores reais de produção necessita, para ser executado, de um conjunto de valores de capital humano obtido dentro de certa razoabilidade. No entanto, é reconhecida, na literatura, a dificuldade de avaliação do capital humano na medida que, em geral, ocorrem problemas de coleta, acesso e qualidade dos dados obtidos. Além do mais, há dificuldade no estabelecimento de proxies eficientes que permitam estabelecer uma conexão entre as variáveis que constituem o capital humano e as informações disponíveis [3].
} 
Tanto no processo de se gerar os dados gedanken quanto na validação dos modelos, os parâmetros foram escolhidos como pertencentes aos seguintes intervalos:

$$
\begin{aligned}
& 0<\alpha<1 \\
& 2<A \leq 10 \\
& 0<B \leq 1 \\
& 0<u<1 \\
& 0<N_{o p} \leq 10
\end{aligned}
$$

Na aplicação do algoritmo, os valores iniciais dos parâmetros foram escolhidos como sendo os pontos médios dos intervalos da equação 4.2. A Figura 1 representa os passos na obtenção dos dados na Etapa 1.

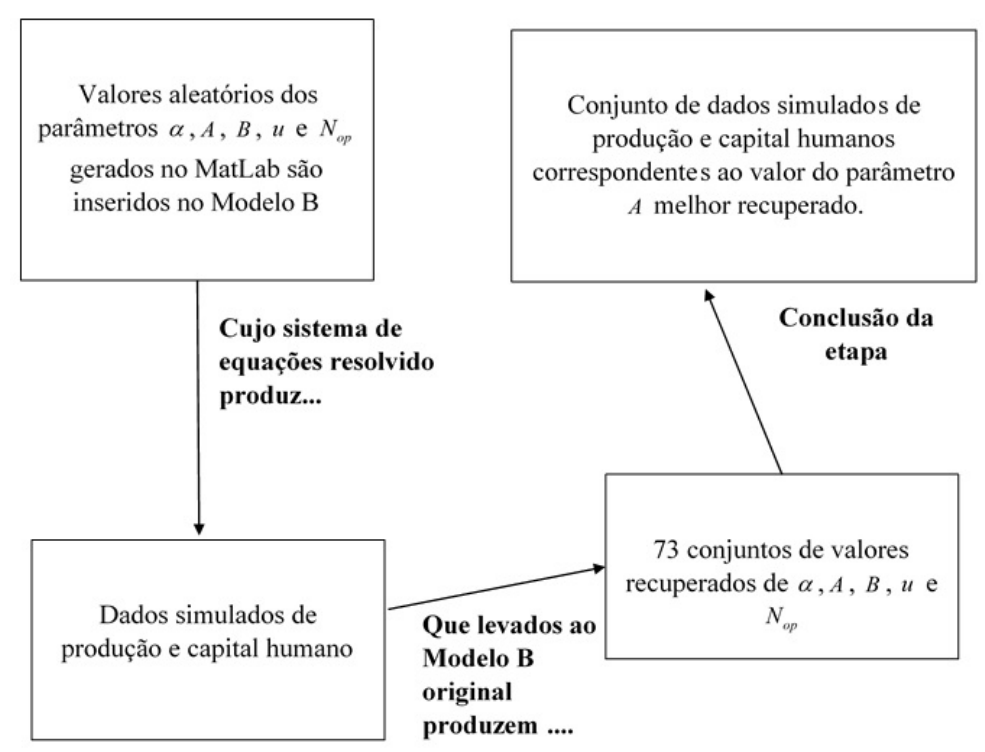

Figura 1: Diagrama que representa os passos da Etapa 1.

\subsubsection{Etapa 2}

A segunda etapa do processo de validação dos modelos utilizou dados de produção de 4 municípios do estado do Rio de Janeiro. Esses municípios foram selecionados de forma a terem uma produção próxima à produção média das microrregiões geográficas onde estão inseridos [14].

Para o ajuste dos modelos a esses municípios, foram considerados os valores do capital humano pertencentes ao conjunto de dados gedanken que forneceu melhor recuperação do parâmetro A, que corresponde ao índice de tecnologia. No caso, foi considerado que todos os municípios apresentam, em média, esses mesmos valores de capital humano, ao longo do tempo. Os dados 
de produção foram obtidos do banco de dados SIDRA do IBGE [15]. Foram considerados 11 anos de produção de mel de cada um dos municípios selecionados, no período de 2001 a 2011.

Ao se verificar se determinado modelo teve um bom ajuste aos dados de produção de certo município, foram dados como entrada no programa desenvolvido no presente trabalho conjuntos de 22 dados: os 11 primeiros dados correspondendo à produção de 2001 a 2011 dos municípios. Os 11 últimos correspondem aos dados gedanken do capital humano recém-mencionado.

Foram considerados bons ajustes de todos os conjuntos de parâmetros recuperados por um dos modelos, que produziram soluções que estavam a uma margem de erro relativo de $30 \%$ dos valores reais de produção dos municípios. Os municípios selecionados foram Itaguaí, Itaocara, Piraí e São Pedro da Aldeia. Foi também aplicado um teste de não decrescimento aos dados de produção ${ }^{7}$. Esse teste foi realizado no sentido de verificar, preliminarmente, se os dados se ajustariam melhor ao Modelo A. Cabe lembrar que esse modelo, por ser mais próximo do modelo neoclássico do qual derivou, tendeu a reproduzir melhor variações de produção não decrescentes.

\section{RESULTADOS E DISCUSSÃO}

A seguir, serão apresentados detalhes da modelagem da produção de mel dos quatro municípios estudados. Dentre estes, foram observadas melhores recuperações com o Modelo B em oito municípios. O Modelo A forneceu melhores ajustes em apenas três casos. Os dois modelos ajustaram-se bem em dois casos. Em cinco casos nenhum dos dois modelos forneceu bons ajustes.

A título de exemplo, serão apresentados quatro casos em que houve bons ajustes de, pelo menos, um dos modelos.

\subsection{São Pedro da Aldeia}

A Tabela 1 mostra os dados de produção e as respectivas recuperações obtidas pelos dois modelos A e B. O teste de não decrescimento da produção mostrou que, em mais de $70 \%$ dos casos, não houve decrescimento da produção em intervalos de tempo consecutivos. Como se pode observar, a média e o desvio padrão dos dados recuperados pelo Modelo A estão bem próximos da média e do desvio padrão dos dados de produção. O que já não acontece com o Modelo B. Todos esses fatores juntos apontam que o Modelo A forneceu o melhor ajuste.

Nas Tabelas a seguir $\sigma$ é o Desvio Padrão.

O gráfico da Figura 2 ilustra o melhor ajuste do Modelo A.

\footnotetext{
${ }^{7} \mathrm{O}$ teste de não decrescimento consistiu, basicamente, na testagem da diferença entre os valores de produção gerados em anos consecutivos. Como saída, ele fornecia o porcentual das diferenças que eram maiores ou iguais a zero.
} 
Tabela 1: Dados de Produção (DP) e Dados Recuperados (DR) dos Modelos A e B, com respectivos erros relativos (ER) para o Município de São Pedro da Aldeia.

\begin{tabular}{cccccc}
\hline Anos & \multicolumn{2}{c}{ Modelo A } & \multicolumn{2}{c}{ Modelo B } \\
\hline & DP & DR & ER & DR & ER \\
\hline 1 & 0.180570 & 0.180570 & 0 & 0.180570 & 0 \\
\hline 2 & 0.823848 & 0.828933 & 0.006172 & 0.378771 & 0.540241 \\
\hline 3 & 1.228838 & 0.914721 & 0.255621 & 0.678764 & 0.447637 \\
\hline 4 & 1.227106 & 0.997565 & 0.187059 & 0.347307 & 0.716970 \\
\hline 5 & 0.196717 & 1.080114 & 4.490700 & 0.102661 & 0.478126 \\
\hline 6 & 1.151950 & 1.164012 & 0.010470 & 0.376344 & 0.673297 \\
\hline 7 & 0.968812 & 1.250409 & 0.290662 & 0.077777 & 0.919719 \\
\hline 8 & 1.502366 & 1.340196 & 0.107943 & 0.411348 & 0.726199 \\
\hline 9 & 1.594964 & 1.434108 & 0.100852 & 1.067713 & 0.330572 \\
\hline 10 & 2.126529 & 1.532797 & 0.279202 & 2.133444 & 0.003251 \\
\hline 11 & 1.297213 & 1.636859 & 0.261827 & 0.957929 & 0.261548 \\
\hline Média & 1.118083 & 1.123662 & & 0.610238 & \\
\hline$\sigma$ & 0.573248 & 0.403665 & & 0.599813 & \\
\hline
\end{tabular}

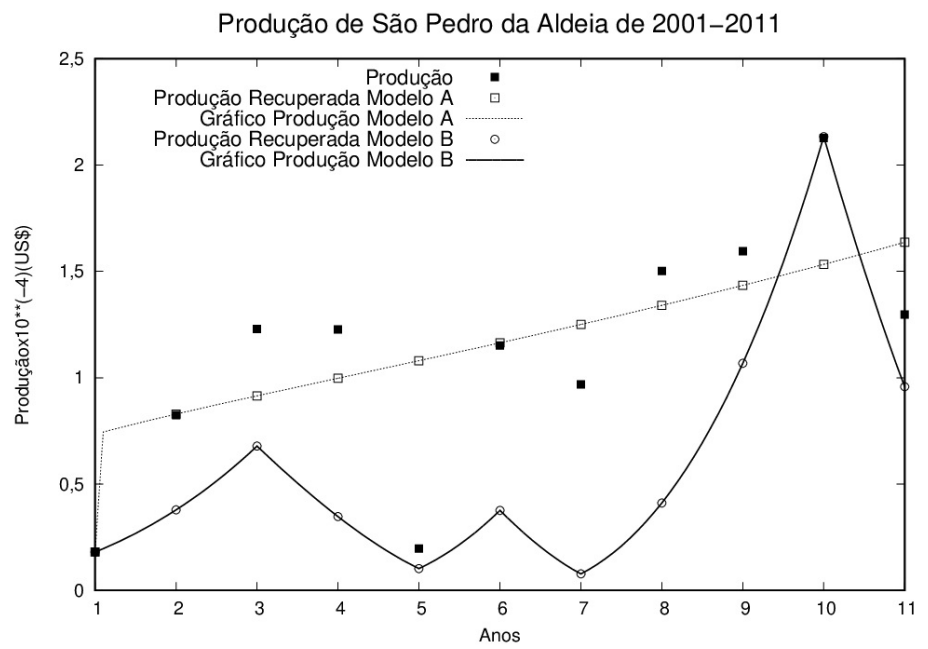

Figura 2: Ajustes obtidos para o município de São Pedro da Aldeia.

\subsection{Itaguaí}

O município de Itaguaí apresentou os seguintes resultados relativos aos Modelos A e B e seus ajustes, Tabela 2 . 
Tabela 2: Dados de Produção (DP) e Dados Recuperados (DR) dos Modelos A e B, com respectivos erros relativos (ER) para o Município de Itaguaí.

\begin{tabular}{cccccc}
\hline Anos & \multicolumn{2}{c}{ Modelo A } & \multicolumn{2}{c}{ Modelo B } \\
\hline & DP & DR & ER & DR & ER \\
\hline 1 & 0.626353 & 0.626353 & 0 & 0.626353 & 0 \\
\hline 2 & 0.768925 & 0.690634 & 0.101817 & 0.727003 & 0.054519 \\
\hline 3 & 1.039786 & 0.759260 & 0.269791 & 0.839494 & 0.192627 \\
\hline 4 & 1.106407 & 0.832421 & 0.247635 & 0.839494 & 0.127821 \\
\hline 5 & 0.740970 & 0.910317 & 0.228547 & 0.832395 & 0.123386 \\
\hline 6 & 0.831964 & 0.993155 & 0.193747 & 0.972390 & 0.168788 \\
\hline 7 & 0.878499 & 1.081150 & 0.230679 & 1.129218 & 0.285395 \\
\hline 8 & 1.216201 & 1.174528 & 0.034264 & 1.304528 & 0.072625 \\
\hline 9 & 1.215210 & 1.273521 & 0.047985 & 1.119299 & 0.078925 \\
\hline 10 & 1.476756 & 1.378372 & 0.066621 & 1.314870 & 0.109622 \\
\hline 11 & 1.534508 & 1.489332 & 0.029439 & 1.534400 & 0.000000 \\
\hline Média & 1.039598 & 1.019004 & & 1.033176 & \\
\hline$\sigma$ & 0.300801 & 0.286347 & & 0.2768154 & \\
\hline & & & & &
\end{tabular}

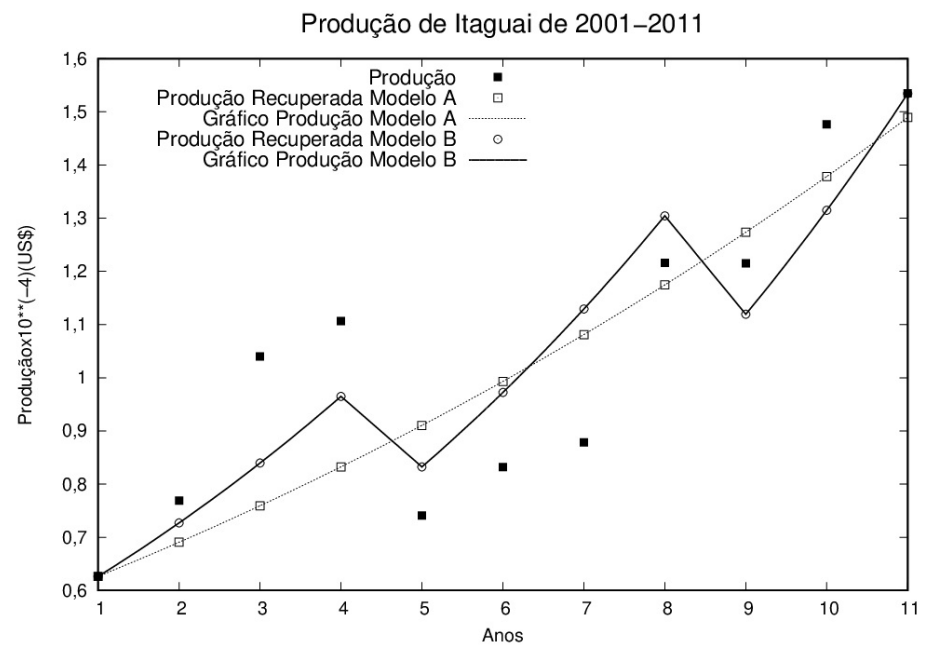

Figura 3: Ajustes obtidos pelos modelos para o município de Itaguaí.

Nesse caso, o teste de não decrescimento mostrou, como no caso anterior, que em mais de $70 \%$ dos intervalos houve não decrescimento da produção, o que sugeriria um bom ajuste do Modelo A. No entanto, ambos os municípios obtiveram médias e desvios padrão próximos a esses mesmos valores obtidos para a produção. Isso indica que, na realidade, ambos os modelos obtiveram um bom ajuste para os valores de produção desse município. Isso está ilustrado na Figura 3. 


\subsection{Piraí}

No caso desse município, o Modelo B forneceu um ajuste melhor que o Modelo A. Isso pode ser ilustrado observando-se a Tabela 3 .

Tabela 3: Dados de Produção (DP) e Dados Recuperados (DR) dos Modelos A e B, com respectivos erros relativos (ER) para o Município de Piraí.

\begin{tabular}{cccccc}
\hline Anos & \multicolumn{2}{c}{ Modelo A } & \multicolumn{2}{c}{ Modelo B } \\
\hline & DP & DR & ER & DR & ER \\
\hline 1 & 2.116060 & 2.116060 & 0 & 2.116060 & 0 \\
\hline 2 & 3.099502 & 2.268530 & 0.268098 & 2.867488 & 0.074855 \\
\hline 3 & 3.906287 & 2.472101 & 0.367148 & 2.867488 & 0.065507 \\
\hline 4 & 3.419805 & 2.733617 & 0.200651 & 2.866551 & 0.161779 \\
\hline 5 & 2.045866 & 3.058736 & 0.495081 & 2.114038 & 0.033321 \\
\hline 6 & 2.687884 & 3.452760 & 0.284564 & 2.865370 & 0.066032 \\
\hline 7 & 3.001347 & 3.921591 & 0.306610 & 3.648201 & 0.215521 \\
\hline 8 & 4.576493 & 4.472449 & 0.022734 & 4.457576 & 0.025984 \\
\hline 9 & 5.101353 & 5.114330 & 0.002543 & 5.289998 & 0.036979 \\
\hline 10 & 6.248748 & 5.858288 & 0.062486 & 6.142853 & 0.016946 \\
\hline 11 & 7.021722 & 6.717645 & 0.043305 & 7.014106 & 0.001084 \\
\hline Média & 3.929551 & 3.835100 & & 3.912057 & \\
\hline$\sigma$ & 1.640778 & 1.538885 & & 1.633150 & \\
\hline
\end{tabular}

No caso desse município, deve-se observar que a média e o desvio padrão do Modelo B estão quase idênticos a esses mesmos valores obtidos para a produção. É importante notar que a média e o desvio padrão obtidos para o Modelo A têm uma pequena discrepância em relação aos valores correspondentes de produção. Isso indica que o ajuste obtido pelo Modelo A não foi ruim. No entanto, como a média e o desvio padrão do Modelo B estão mais próximos da média e o desvio padrão para a produção, o ajuste do Modelo B foi considerado melhor.

O teste de não decrescimento indicou o bom ajuste do Modelo A, pois os intervalos de não decrescimento da produção foram da ordem de $82 \%$. A Figura 4 ilustra esses fatos.

\subsection{Itaocara}

Para o município de Itaocara, os dados de produção e os ajustes do Modelo A e do Modelo B produziram os seguintes resultados, Tabela 4. Para esse município, a média e o desvio padrão da produção gerada pelo Modelo A estão mais próximos dos valores correspondentes dos dados reais de produção. No entanto, se observarmos o coeficiente de variação (razão entre o desvio padrão e o valor absoluto da média) para os dados gerados pelo Modelo A, ele é da ordem de 33,9\%. Já os dados gerados pelo Modelo B produzem um coeficiente de variação da ordem de $48,5 \%$. Este último está bem próximo do mesmo coeficiente relativo aos dados de produção, que 
Produção de Pirai de 2001-2011

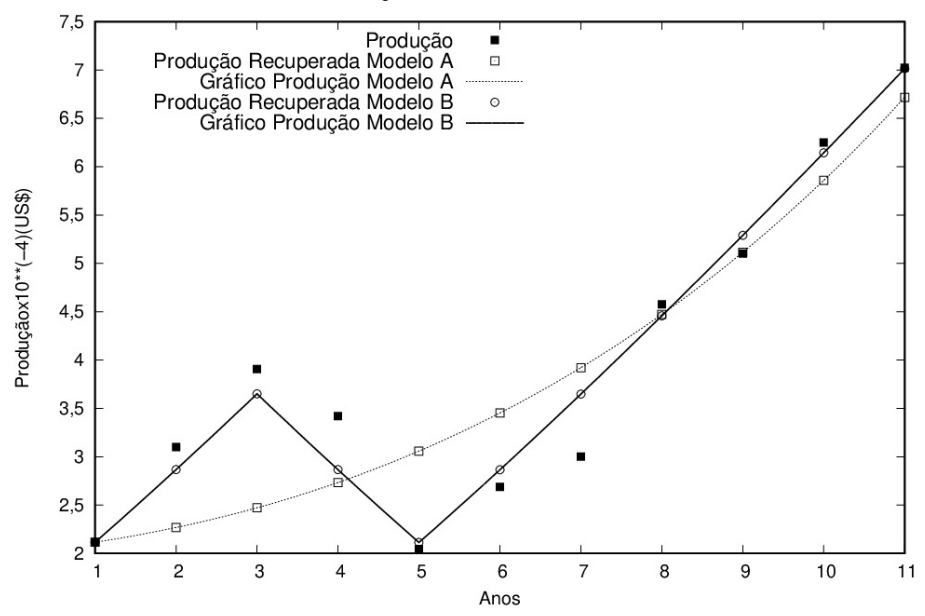

Figura 4: Ajustes obtidos pelos modelos para o município de Piraí.

Tabela 4: Dados de Produção (DP) e Dados Recuperados (DR) dos Modelos A e B, com respectivos erros relativos (ER) para o Município de Itaocara.

\begin{tabular}{cccccc}
\hline Anos & \multicolumn{2}{c}{ Modelo A } & \multicolumn{2}{c}{ Modelo B } \\
\hline & DP & DR & ER & DR & ER \\
\hline 1 & 1.083423 & 1.083423 & 0 & 1.083423 & 0 \\
\hline 2 & 1.647697 & 1.158193 & 0.297084 & 1.254384 & 0.056980 \\
\hline 3 & 2.835780 & 1.252891 & 0.558847 & 1.476390 & 0.229796 \\
\hline 4 & 1.609320 & 1.369095 & 0.149271 & 1.251973 & 0.049306 \\
\hline 5 & 0.786871 & 1.508039 & 0.916501 & 1.078798 & 0.137639 \\
\hline 6 & 1.279945 & 1.670906 & 0.305452 & 1.250998 & 0.000511 \\
\hline 7 & 1.313643 & 1.859094 & 0.415220 & 1.475269 & 0.015138 \\
\hline 8 & 1.716989 & 2.074375 & 0.208147 & 1.776709 & 0.001209 \\
\hline 9 & 1.898766 & 2.318992 & 0.221315 & 2.198423 & 0.024906 \\
\hline 10 & 2.421880 & 2.595699 & 0.071771 & 2.820270 & 0.027059 \\
\hline 11 & 3.986559 & 2.907791 & 0.270601 & 3.805946 & 0.002053 \\
\hline Média & 1.870988 & 1.799864 & & 1.770235 & \\
\hline$\sigma$ & 0.911499 & 0.609982 & & 0.857760 & \\
\hline
\end{tabular}

é da ordem de 48,7\%. Esse dado atesta que o Modelo B obteve um melhor ajuste que o Modelo A. 
Assim como no caso do município anterior, o teste de não decrescimento mostrou uma taxa de $82 \%$ de intervalos em que a produção foi não decrescente em anos consecutivos. Apesar da taxa elevada de intervalos de não decrescimento da produção, foi o Modelo B que se ajustou melhor aos dados de produção, como pode-se observar na Figura 5.

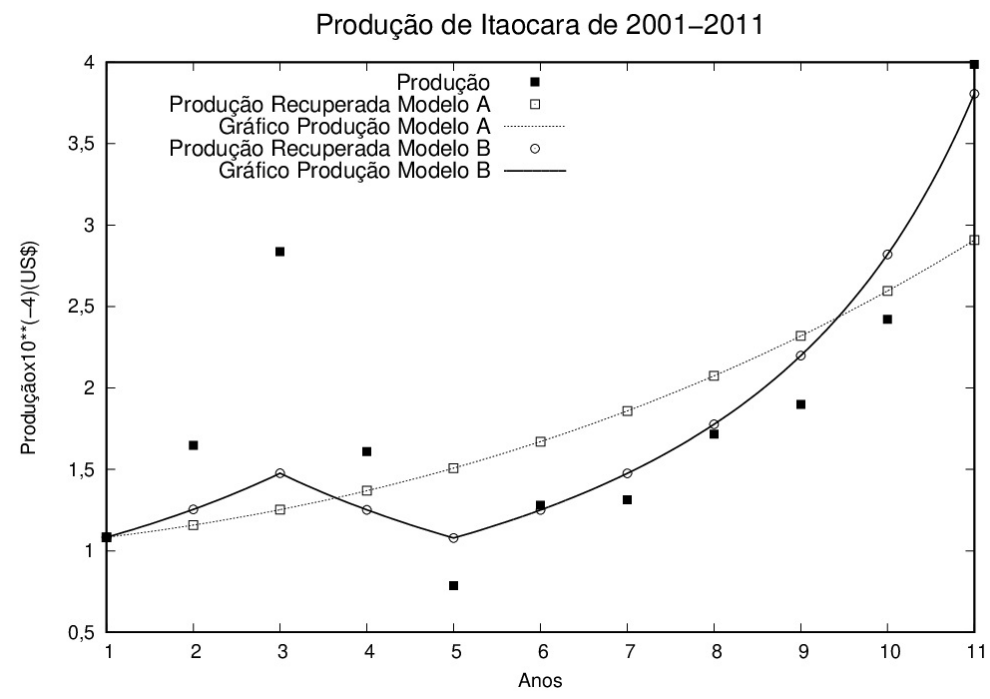

Figura 5: Ajustes obtidos, por ambos os modelos, para a produção de Itaocara.

\subsection{Contribuição para a pesquisa}

Uma das contribuições do presente trabalho é a possibilidade de obtenção do índice de tecnologia de uma determinada localidade como parâmetro de ajuste dos modelos, por um período de onze anos. Os valores desses parâmetros permitem ordenar os municípios segundo a sua produtividade na produção de mel. Este seria um elemento importante para a tomada de decisões do gestor do agronegócio, contribuindo para, por exemplo, a alocação de recursos em municípios que se mostrassem menos produtivos em certo período.

No caso dos municípios tomados como exemplo, a Tabela 5 os estratifica, do mais produtivo ao menos produtivo, segundo os valores obtidos para o parâmetro A dos modelos. Esses parâmetros correspondem ao índice de tecnologia dessas localidades, por onze anos.

\section{CONCLUSÕES}

O presente artigo traz à luz, mais uma vez, a importância da modelagem no tratamento de aspectos complexos da realidade. Utilizando-se apenas métodos numéricos para o ajuste dos modelos aos dados de produção de quatro municípios do Rio de Janeiro, agregando-lhes dados simulados de capital humano (veja a seção 4.2), conseguiu-se obter um perfil da produção nessas localidades. 
Tabela 5: Índice de tecnologia dos municípios.

\begin{tabular}{cc}
\hline Município & Índice de Tecnologia A \\
\hline São Pedro da Aldeia & 9.319440 \\
\hline Piraí & 6.079417 \\
\hline Itaguaí & 5.998481 \\
\hline
\end{tabular}

Como se sabe. a produção de mel é extremamente dependente de fatores ambientais. Esses fatores podem inserir uma certa aleatoriedade no delineamento da produção. Tudo indica que o peso desses fatores aleatórios foram mitigados, em certa medida, pela incorporação da variável capital humano - isto é, a formação e o incremento nas habilidades do produtor - nos modelos desenvolvidos.

De grande importância também foi a contribuição dada à determinação, por meio de um método alternativo, do índice de tecnologia de uma certa localidade, durante um período de onze anos. Não haverá dificuldade em adequar-se esse intervalo de tempo para um período mais conveniente, deste que é um indicador importantíssimo para o gestor do agronegócio.

\begin{abstract}
In this work, mathematical models were developed which allow the simulation of the honey production at a certain municipality. These models are based in ordinary differential equations and were based on the neoclassical macroeconomic model by Lucas [23] for macroeconomic growth. In the models' parameters determination, nonlinear numeric optimization methods were employed. As a result of the numerical solution of the model, honey production from four municipalities was simulated. It was also obtained the technology level attained by the honey production of a certain place, by a period of eleven years. Through this index it is possible to order the municipalities in terms of technology application. This is one of the reasons why this index is an indicator of vital importance for agribusiness management.
\end{abstract}

Keywords: Lucas model, mathematical models, honey production.

\title{
REFERÊNCIAS
}

[1] D. Acemoglu. A Microfoundation for social increasing returns in human capital accumulation. The Quarterly Journal of Economics, 111(3) (1996).

[2] M.B. Arouxet, N. Echebest \& E.A. Pilotta. Active Set Strategy in Powell's Method for Optimization without Derivatives. Computational and Applied Mathematics, 30(1) (2011), 171-196.

[3] R.A. Arraes, F.Z. Mariano \& S.J.A. Barros. Medidas de Capital Humano e seus efeitos sobre os Diferenciais de Produtividade: uma comparação entre os estados do Ceará e Santa Catarina. In E.B.S. Carvalho, M.C. Holanda \& M.P. Barbosa (editors), "Economia do Ceará em Debate”. IPECE, Fortaleza (2006), pp. 123-141. 
[4] W.F. Barbosa \& E.P. Sousa. Desempenho Competitivo dos Apicultores Fixos e Migratórios na Microrregião do Cariri. Revista de Economia e Administração, 11(1) (2012), 5-27.

[5] S.J. Chapman. "Fortran 95/2003 for Scientists and Engineers". McGraw-Hill (2007).

[6] M.D. Cox \& M.R. Myerscough. A Flexible Model of Foraging by a Honey Bee Colony: the Effects of Individual Behaviour on Foraging Success. Journal of Theoretical Biology, 223 (2003), 179-197.

[7] R. Dathein. "O crescimento do desemprego nos países desenvolvidos e sua interpretação pela teoria econômica: as abordagens neoclássica, keynesiana e schumpeteriana”. Ph.D. thesis, Instituto de Economia, UNICAMP, Campinas, SP (2000).

[8] G. DeGrandi-Hoffman, F. Ahumada, R. Curry, G. Probasco \& L. Schantz. Population growth of Varroa destructor (Acari: Varroidae) in commercial honey bee colonies treated with beta plant acids. Experimental Applied Acarology, 2 (2014), 171-186.

[9] Z. Dogan, M. Karagoz \& G.O. Ozbakir. Long Years Apiculture Data Model of Turkey: An Econometric Time Series Analysis. The Journal of Animal and Plant Sciences Analysis, 24(5) (2014), 1573-1578.

[10] A. Evangelista-Rodrigues, D.N. Souza, G.R.B. Cruz, M.L. Rodrigues, M.C. Silva \& G.C. Gois. Modelos Matemáticos Aplicados no Crescimento de Colmeias de Apis Mellifera L. no Cariri Paraibano. Archivos de Zootecnia, 60(229) (2011), 137-140.

[11] M.K.A. Gavina, J.F. Rabajante \& C.F. Cervancia. Mathematical Programming Models for Determining the Optimal Location of Beehives. Bulleting of Mathematical Biology, 76 (2014), 997-1016.

[12] A. Golinsky. "Avaliação da viabilidade econômica e nível tecnológico da apicultura no estado do Rio de Janeiro". Ph.D. thesis, Centro de Ciências e Tecnologias, Universidade Estadual do Norte Fluminense Darcy Ribeiro, Campos dos Goytacazes, RJ (2009).

[13] L.S. Gonçalves. 50 anos de abelhas africanizadas no Brasil. In "Anais do $16^{\circ}$ Congresso Brasileiro de Apicultura”. CBA (2006), pp. 14-16.

[14] IBGE. "Divisão Regional do Brasil em Mesorregiões e Microrregiões Geográficas". Diretoria de Geociências, Rio de Janeiro (1990).

[15] IBGE. "Banco de Dados Agregados". http://sidra.ibge.gov.br (2017).

[16] C.H.A. Irgoin. Simulación del Modelo de Solow-Swan. Contribuciones a la Economia: Revista Academica Virtual, (2011).

[17] T.R. Jain \& V.K. Ohri. "Introductory Macroeconomics”. ed. New Delhi (2010).

[18] E.O. Jatulan, J.F. Rabajante, C.G.B. Banaay, A.C.F. Jr. \& E.C. Jose. A Mathematical Model of Intra-Colony Spread of American Foulbrood in European Honeybees (Apis mellifera L.). PLOS-One, 12(10) (2015). 
[19] A.S. Khan, V.D. Matos \& P.V.P.S. Lima. Desempenho da Apicultura no Estado do Ceará: Competitividade, Nível Tecnoloógico e Fatores Condicionantes. Revista de Economia e Sociologia Rural, 47(3) (2009), 651-675.

[20] D.S. Khoury, A.B. Barron \& M.R. Myerscough. Modelling Food and Population Dynamics in Honey Bee Colonies. PLOS-One, 8(5) (2016).

[21] A.S. Koshiyama \& et al. Proposta de Diagnose Apícola, de sua Tecnologia e Sanidade por meio do Indicador Idapi. Revista Portuguesa de Ciências Veterinárias, 209(591-592) (2014), 94-99.

[22] M.C. Lorenzon. "Depoimento recebido por email, pelos autores em 11/08/2017" (2017).

[23] R.E. Lucas. On the Mechanics of Economic Development. Journal of Monetary Economics, 22 (1988), $3-42$.

[24] N.G. Mankiw, D. Romer \& D. Weil. A Contribution to the Empirics of Economic Growth. The Quarterly Journal of Economics, 107 (1992), 407-438.

[25] V.D. Matos \& S.J. Freitas. Uma Estimativa dos Fatores Condicionantes do Nível Tecnológico dos Apicultores de Limoeiro do Norte: Uma Apicação do Modelo Probit. In Sober (editor), "Congresso da Sober", volume 43. Sober, Ribeirão Preto/SP (2005).

[26] E.S. Monteiro \& et al. Inovação e Tecnologia no Arranjo Produtivo de Apicultura da Nordeste Paraense. Revista de Política Agrícola, (4) (2014).

[27] M.J.D. Powell. The NEWUOA Software for Unconstrained Optimization without Derivatives. In G.D.D. Pillo \& M.D. Roma (editors), "Large Scale Optimization”. Springer, New York (2006), pp. 255-297.

[28] C.M. Rowland \& A.R. McLellan. A Simple Mathematical Model of Brood Production of Honeybee Colonies. Journal of Apiculture Research, 21(3) (1982), 157-160.

[29] G.E. Silva Filho \& E.B.S. Carvalho. A teoria do crescimento endógeno e o desenvolvimento endógeno regional: investigação das convergências em um cenário pós-cepalino. Revista Econômica do Nordeste, 32 (2001), 467-482.

[30] D.J.T. Sumpter \& S.J. Martin. The dynamics of virus epidemics in Varroa-infested honey bee colonies. Journal of Animal Ecology, 73 (2004), 51-63.

[31] H. Uzawa. Optimum Technical Change in an Aggregative Model of Economic Growth. International Economic Review, 6(1) (1965), 18-31.

[32] H.R. Varian. "Microeconomia: uma Abordagem Moderna”. Elsevier Campus (2012).

[33] M. Wilhelm, M. Chhetri, J. Rychtár \& O. Rueppell. A Game Theoretical Analysis of the Mating Sign Behaviour of the Honey Bee. Bulleting of Mathematical Biology, 73 (2011), 623-638. 